\title{
Genetic analysis of multiple sclerosis in Orkney
}

\author{
DEREK F. ROBERTS AND MARY J. ROBERTS \\ From the Department of Human Genetics, University of Newcastle upon Tyne
}

DAVID C. POSKANZER

From the Neurology Service, Massachusetts General Hospital, Boston

SUMMARY In a family study of all patients with multiple sclerosis (MS) in Orkney, the number of inbred among patients, although high for Britain, is not higher than the number among controls, and the inbreeding coefficients appear to eliminate recessive involvement of rare genes from the aetiology. The kinship coefficients show that the ancestries of patients and controls are closely enmeshed, and eliminate from the aetiology involvement of recently introduced genes dominant or codominant in effect. Family histories show that single locus inheritance is unlikely unless penetrance is very low. Multifactorial genetic involvement is much more likely, and it is compatible with all recent findings; on this hypothesis heritability estimates, not altogether satisfactory because of the limited number of patients in the population, suggest that the genetic contribution to the aetiology of the disease in Orkney is only moderate.

Several features of the population of Orkney make it particularly suitable for an attempt at genetic analysis of multiple sclerosis (MS), which there attains its highest reported prevalence. These features include the geographically well-defined area inhabited, the extent and accuracy of the vital records, and the strong sense of identity and relative constancy of the population; for although there has been a steady flow of emigrants, relatively few recent immigrants have settled and contributed to the gene pool of the population. Over the years there has been intermittent discussion of the role of genetic factors in MS which has gained new impetus from the demonstration of associations of MS with various histocompatibility antigens, but there have been no recent efforts to attempt a population genetic analysis of the disorder. This was the object of the present study.

\section{Material and methods}

All patients with MS in the Orkney Islands alive on 1 December 1974 were identified, and two more were included in whom the diagnosis was confirmed in 1976; in two cases the family doctor requested that the family not be visited, so these were omitted. This gave a total of 51 patients, 45 of whom were diagnosed as probable cases and six as possible cases, according to established international criteria; 48 had been born in Orkney, three elsewhere. For each Orkney-born patient a contiguous control was selected who was not a member of the same immediate family, who was born in the same parish in the same year, was of the same sex, had lived in the same area for the first 15 years of his life, and was not affected. Where a control was subsequently himself found to have a neurological disorder, or to be a first or second degree relation of the patient to whom he was matched, a replacement was obtained. Each patient, and each control, was interviewed to obtain a family history, including the necessary identification of his parents, their dates and places of birth, the maternal maiden name, and relevant details of the immediate relatives, so that a comprehensive family history of three or four generations was obtained. This was verified from documentary sources and ancestry was then traced further back for each subject through vital records, parish records, electoral returns, land deeds, and the wide variety of documentation that is available for this type of genetic investigation. The same procedure was followed for the 51 patients and the 48 contiguous controls. These are the subjects for whom HLA and other serological findings are reported (Poskanzer $e t$ al., 1980; Roberts et al., 1979); no controls were included for the three patients from outside Orkney. In all, 13453 record searches were completed, relating to 4327 individuals traced back to an 
arbitrary baseline taken as 1775 . From these data the intricate web of relationships was established, consanguinity and kinship measured, and pedigree analysis undertaken.

\section{Results}

\section{CONSA NG UINITY}

It has long been known that, in a recessive disorder, the proportion of patients occurring in offspring of first-cousin unions is higher than in offspring of unrelated parents; the elevation increases with increasing rarity of the condition and decreasing frequency of consanguineous unions in the population. ${ }^{*}$ The proportion of cousin unions is low in the British Isles, so that consanguinity may be expected to provide a valuable pointer towards a recessive component in a given disorder. This effect is due to the fact that an offspring of a consanguineous union is inbred: both the genes that he possesses at a given locus, one from his father and one from his mother, may be replicates of a single gene present in their common ancestor, and therefore identical by descent. The closer the consanguinity of the parents, the greater the probability that their offspring will be an identical homozygote at a given locus. Biological inbreeding, the effect of consanguinity, can be quantified by the inbreeding coefficient + of an individual, which measures the probability that both the genes he possesses at a given locus are identical by descent or, expressed in a different way, the proportion of loci at which he is expected to be an identical homozygote. In the absence of more remote consanguinity, the inbreeding coefficient of the offspring of a first-cousin union is 0.0625 , and of a brother/sister incestuous union $0 \cdot 25$. A population can therefore be characterised by the mean and distribution of inbreeding coefficients of its members. If, in a section of the population identified by a particular disorder, the distribution of inbreeding coefficients is displaced upwards and has a higher mean than that in unaffected individuals, then it is a reasonable inference that genes recessive in their effect contribute to that disorder.

The result for the MS patients in Orkney came as something of a surprise. Of the 51 patients, six (11.8\% of all patients, $12.5 \%$ of the Orkney-born)

\footnotetext{
$* k=\frac{c(1+15 q)}{16 q+c(1-q)}$

where $k$ is the proportion of patients born to first-cousin parents, $c$ is the incidence of first-cousin unions in the general population, and $q$ is the gene frequency (Dahlberg, 1948).

$+\mathbf{F}=\mathbf{\Sigma}\left[(\mathbf{(})^{\mathrm{n}}\left(\mathbf{1}+\mathbf{F}_{\mathrm{A}}\right)\right]$

where $F$ is the inbreeding coefficient of the subject, $F_{A}$ is the inbreeding coefficient of the common ancestor, $n$ is the number of zygotes in a connecting path through the common ancestor including both parents of the subject, and summation is over all such paths over all common ancestors.
}

had positive inbreeding coefficients, that is, they were inbred to some extent. There are no directly similar data with which this finding can be compared, but indirect evidence indicates that this figure is considerably higher than expected for a population in the British Isles. In the Republic of Ireland in 1959-68 the first-cousin marriage rate among Catholics was $0.16 \%$ and in Northern Ireland it was $0.06 \%$ (Masterson, 1973). Of all consanguineous unions, first-cousin marriages represent only a proportion, so the extent of inbreeding in the population would be underestimated if they alone were considered (Roberts, 1969); if for the sake of argument this proportion is taken to be as extremely low as one-tenth or one-twentieth, and the Irish consanguinity estimates are adjusted accordingly, they would remain greatly below that observed in the Orkney patients.

In the Orkney controls, however, a similar figure emerges-there are five inbred individuals among the 48 examined $(10 \cdot 4 \%)$. It appears, therefore, that the elevated incidence of inbred individuals, observed in MS patients in Orkney, is a feature of the population as a whole rather than of any association with the disease.

The inbreeding coefficients, however, suggest a slight difference, in that the distribution of these coefficients may be shifted slightly upwards in the patients. There are two pointers to this. Firstly, in the 51 patients the mean inbreeding coefficient is $0.00239(0.00254$ in the Orkney-born $)$, and in the controls it is 0.00118 , approximately half as high. In those who are inbred, the mean in the patients $(0.02035)$ is again higher than in the controls $(0 \cdot 01133)$. Secondly, two of the patients, but no control, have coefficients greater than 0.03 . Unfortunately the numbers of subjects are insufficient for the significance of this suggestion to be meaningfully tested.

Comparison has to be restricted to the present samples because there is no information on general inbreeding levels in Orkney, and the few modern studies that provide comparative population data are primarily related to small isolated populations such as the inhabitants of Tristan da Cunha, with a mean inbreeding coefficient of 0.043 , or the Hutterites, $0 \cdot 022$. By comparison with these, the Orcadian samples of patients and controls are not highly inbred, so that widespread more severe effects of close inbreeding are not to be expected.

\section{KINSHIP}

If a new gene dominant or codominant in effect arrives in a population and is transmitted by the introducer to his descendants over several generations, one would expect the coefficient of 
kinship between all affected individuals to be greater than those between affected and all non-affected members in the population. The coefficient of kinship $(\varnothing)^{*}$ of two individuals measures the probability that two homologous genes drawn at random, one from each individual, will be identical. For a brother and sister the kinship coefficient is 0.25 ; for first cousins it is 0.0625 . Examination of the kinship coefficients in a series of patients will therefore indicate the validity of the hypothesis that a condition is due to a single gene, dominant or codominant in effect, of recent introduction.

In the Orkney material (Table 1), comparing each of the 51 patients with every other gives 1275 possible paired comparisons and hence 1275 kinship coefficients $\emptyset$. For the 48 Orkney-born patients there are 1128 , and among the 48 controls 1128 such pairs also. The number of possible comparisons between every patient and every control is 2448 , and between every Orkney-born patient and control 2304. The mean kinship coefficient among all patients is 0.000344 and among the Orkney born 0.000387 ; the mean among controls is similar at 0.000463 . In the total patient comparisons there are 1224 zero coefficients and 51 non-zero, and in the Orkney-born 1078 and 50; the means of the non-zero coefficients are 0.008607 and 0.008741 respectively. Among the controls there are 1089 zero coefficients and the

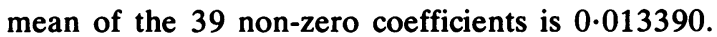
The difference between the patient set and the control set in the numbers of positive coefficients is not significant. Only 10 of the Orkney-born patients are not related in some way to at least one of the others, and only 14 of the controls are not related to some other control. It appears that while there is an intricate web of relationships among patients and a similar web of relationships among controls, there is no reason to believe that the patients are more closely related to each other than are the controls to each other.

Of the 2448 possible comparisons between all patients and controls, there are 2367 zero

${ }^{\omega_{A B}}=(\xi)^{n+p+1}\left(1+F_{C}\right)$

where $\mathrm{n}$ is the number of zygotes in the path connecting $\mathrm{A}$ and the common ancestor $(C), p$ the number in that connecting $B$ and $C, F_{C}$ is the inbreeding coefficient of the common ancestor, and summation is over all paths over all common ancestors.

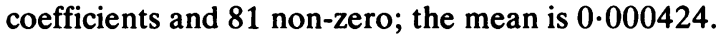
Of the possible 2304 comparisons involving Orkney-born, the corresponding figures are 2227, 77 , and 0.000419 . The means of the non-zero coefficients are 0.012810 and 0.012537 respectively. Again, the proportion of non-zero coefficients is not significantly different from that within either patients or controls. Of the total number of 99 subjects, only 14 are not related to at least one other over the 10 generations that were investigated, and of these 14 , two are among the patients born outside Orkney. There is thus no reason to regard the affected as being different in their ancestry from the controls, and both sets of families are seen to be inextricably intertwined together. It is extremely unlikely, therefore, that a recently introduced single dominant or codominant gene is responsible for the disease.

\section{FAMILY HISTORIES}

(a) Monogenic inheritance

For any single-gene mode of inheritance the family histories of Orkney patients are remarkably empty of affected relatives. However, the occurrence of the disease in relatives of different degrees shows a characteristic gradient. There are 258 first-degree relatives of the patients, of whom three are affected. Of these, two of the cases occurred among the 94 parents, and one among the 164 sibs. Among second-degree relatives, four out of 893 were affected, and among third-degree relatives three out of 825 . The proportions are 1 in 86 for first-degree relatives, 1 in 223 for second-degree, and 1 in 275 for third-degree. The closer the degree of relationship, the greater the proportion of relatives affected. Although for first-degree relatives overall the proportion is similar to that in other studies, in a small sample of this size it is not surprising that the proportions of 1 in 47 parents and 1 in 164 sibs appear to reverse the general figures observed in the literature (1 in 175 and 1 in 96 respectively). These figures are considerably in excess of the cases among the relatives of the controls, one out of 329 first-degree, 0 out of 829 second-degree, and one out of 801 third-degree.

Of the 51 patients' pedigrees, there is none that suggests simple dominant, recessive, or $x$-linked

Table 1 Kinship coefficients

\begin{tabular}{lllllc}
\hline & $\begin{array}{l}\text { Number of } \\
\text { comparisons }\end{array}$ & $\begin{array}{l}\text { Number of } \\
\text { unrelated } \\
\text { individuals }\end{array}$ & $\begin{array}{l}\text { Zero } \\
\text { coefficients }\end{array}$ & $\begin{array}{l}\text { Non-zero } \\
\text { coefficients }\end{array}$ & $\begin{array}{l}\text { Mean } \\
\text { non-zero } \\
\text { coefficients }\end{array}$ \\
\hline Control $\times$ control & 1128 & 14 & 1089 & 39 & 0.000463 \\
Total affected $\times$ affected & 1275 & 12 & 1224 & 51 & 0.000344 \\
Orkney-born affected $\times$ affected & 1128 & 10 & 1078 & 50 & 0.000387 \\
Total affected $\times$ control & 2448 & 14 & 2367 & 8.008607 & 0.000424 \\
Orkney-born affected $\times$ control & 2304 & 12 & 2227 & 77 & 0.000419 \\
\hline
\end{tabular}


inheritance. Although there are three in which a first-degree relative is affected, all other affected relatives occur at such a distance from the propositus as to prove that if a dominant or codominant gene is operative, environmental or other genetic factors must be important enough to prevent its manifestation in the majority of those who carry it. From segregation information in the present data, penetrance can be estimated at $12 \%$. Taken as a whole, the pedigree data do not support the hypothesis of a simple recessive disorder, for of the 164 sibs of the 51 patients only one is affected, far too few on this hypothesis. The occurrence of so many affected females (30 females and 21 males) eliminates sex-linked recessives.

\section{(b) Multifactorial}

There are many diseases in which there is a definite familial tendency but in which the mode of inheritance is not Mendelian, and it is the pattern shown by the family histories of these that the MS data most resemble. It is becoming increasingly clear that in these conditions a different type of inheritance occurs, such as that which applies to continuously varying characters like stature. Liability to develop the disease is regarded as being under the control of a system of polygenes situated at a number of loci and interacting with each other. Some genes promote liability to the disease, their alleles the reverse, just as some genes promote tall stature and their alleles short. The liability of an individual to develop the disease depends on the number of genes promoting it that he possesses, so that in the ideal case where alleles are equal in effect, the genotypic liability in the population is distributed in a Gaussian curve. Towards one end of the distribution there occurs a threshold. Individuals who possess the appropriate loading of genes for susceptibility fall beyond this threshold and develop the disease. But just as stature can be influenced by differences in nutrition during growth, and some individuals who would genetically be near the threshold are thrust across it by their environment, so also environment may affect the position of an individual relative to the threshold for MS; all the factors thought to affect the development of the condition, for example, diet, infection, latitude, climate, would affect the position of the individual relative to the threshold. On such a model can be calculated the heritability, defined as the proportion of the variance in liability to develop the disease that is due to additive genetic factors. This model can be applied to MS.

Heritability estimates are derived by comparing the incidence of the disorder in relatives of the patients with the incidence in a control sample or in the population at large. The present control sample is of limited applicability, because of the small proportion of relatives of different degrees who are affected, and the only useful data are those for a comparison of first-degree relatives, which provides a heritability estimate of $31 \cdot 1 \% \pm 11 \cdot 7 \%$. This estimate is similar to that obtained by applying the population prevalence, again using first-degree relatives of patients, which gives a figure of $30 \cdot 8 \% \pm 14 \cdot 8 \%$. Using parents alone, comparison with the population prevalence gives the highest heritability estimate of $46 \cdot 6 \% \pm 19 \cdot 3 \%$, which is rather closer to those calculated from the studies of Curtius (1933), Pratt et al. (1951), Millar and Allison (1954), Sutherland (1956), and Schapira et al. (1963). The numbers were, however, too few for calculation of heritabilities to be satisfactory, and estimates from other degrees of relationship are not useful.

\section{Discussion}

Although this represents a total survey of a population with the highest reported prevalence of MS, none the less the numbers of patients and of relatives remain small, so less information than might be hoped for emerges from the genetic analysis. However, a number of points are clear.

\section{CONSANGUINITY}

The possibility that parental consanguinity is an element in the aetiology of MS was first noted by Curtius (1933), who observed that of 106 patients with MS, first-cousin marriages between their parents had occurred in three; this number is seven and a half times that expected from the incidence of first-cousin marriages in the appropriate Prussian control population. Bell (1940), in her general study of the consanguinity rate in the hospital population, observed that for 632 MS patients, consanguineous marriages had occurred in the parents of eight, again in excess of the number (five) expected from the incidence in parents of a general hospital sample. Pratt et al. (1951) reported on data from 134 patients at the Middlesex Hospital, finding three offspring of consanguineous unions, again in excess of the expected number of $1 \cdot 4$. The differences in the excess between the first and the last two series may be attributed perhaps to the fact that Curtius made comparison with the incidence in a normal population, the other authors with a sample selected by hospitalisation. But the trend to increased parental consanguinity is clear and the only data that apparently conflict with it are the present findings, and those of Myrianthopoulos and MacKay (1960) in the United States of America, who found no case of consanguinity in the parents of 54 pairs of twins. 
The present consanguinity findings elucidate three possible hypotheses:

(i) There is recessive involvement of rare genes. This can be eliminated on account of the lack of elevation of the number of inbred patients, or of any pronounced displacement of the distribution of the inbreeding coefficients.

(ii) There is recessive involvement of genes not of low frequency, but widely distributed in the population. This remains a possibility. For example, for a recessive condition of incidence 1 in 300 , and therefore with gene frequency 0.06 , in a population in which $1 \%$ of marriages occur between first-cousins, only $2 \%$ of patients would derive from such unions; if the incidence of the condition were 1 in 200 , then only $1.8 \%$ of patients would so derive. The present findings are compatible with figures of this magnitude. However, this would imply a gene frequency three times as high as that for the most common established recessive disorder in Britian.

(iii) There is no recessive involvement. This hypothesis cannot be eliminated, and requires further data to establish whether or not the difference in the distributions of the inbreeding coefficients is real.

KINSHIP

Again, there are few data with which these coefficients can be compared. In the small isolated populations such as the Pacific atolls of Pingelap and Mokil, the mean kinship in the present inhabitants is about 0.04 and 0.02 respectively (Morton et al., 1971), by comparison with which the Orkney subjects are much less closely related; part of this difference is due to the fact that the Orcadian population is numerically greater and is long established, whereas the atoll populations derive from founders only nine generations ago. The few available estimates of kinship for modern populations, such as those for Switzerland calculated by Morton and Hussels (1970), yielding a mean

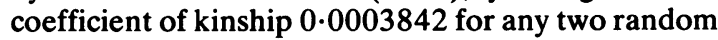
Swiss, derive from indirect methods, so may not be directly comparable with results obtained in the present study by path analysis, but appear of the same order of magnitude.

As regards their interpretation in relation to MS, the results are of direct relevance to hypotheses of single gene control.

(i) There is involvement of genes of dominant or codominant effect of recent introduction. This can be eliminated by the similarity of the matrices of kinship coefficients within patients, within controls, and between patients and controls. (ii) There is involvement of such genes widely distributed, and therefore long established in the population. This remains a possibility, for the amount of interrelationship observed suggests that any gene introduced 20 generations ago, and sufficiently advantageous in effect to become widely distributed through the population, would certainly not have been impeded in so doing. This, however, would imply that such a gene would have some evolutionary advantage. Since this is difficult to predicate, any such gene would need to have been introduced by a remote ancestor who made a major contribution to the gene pool of the subsequent population, possibly in the early days of settlement-the 'founder effect'.

FAMILY HISTORIES AND MODES OFINHERITANCE Monogenic inheritance

Inspection of the pedigrees indicated that single locus inheritance of MS is unlikely. There is indeed little real evidence for dominance or codominance, such as is implied in recent discussions of a 'gene' for MS linked- to the histocompatibility complex. Such a 'gene' seems to have been postulated primarily in an attempt to explain the HLA and related associations that appeared to exist, which the present Orkney study questions (Poskanzer et al., 1978). Perhaps also there was some unintentional bias, for the few family protocols that have been published in the literature relate to nuclear families, and do not show the more extended family which is so necessary for proper interpretation of any apparent familial clustering. Certainly from the present analysis a gene of major effect, dominant or codominant in action, appears highly unlikely. The pedigree analysis indicates that if it exists its effects are outweighed by environmental or other genetic factors. The kinship analysis indicates that if it exists it must have been introduced very early in the history of the population to become as widespread as it is, and certainly not within recent times.

For recessive involvement the evidence is less negative. Certainly recessive involvement of rare genes can be eliminated by the results of the analysis of inbreeding, but not genes recessive in effect widely distributed in a population. With this hypothesis, however, the family analysis does not agree, for if it were so one would expect appreciably more than one out of 164 sibs to be affected. Again, if this is a component in the mode of inheritance, then the effect of such genes must be heavily obscured by environmental factors.

Sex-linkage can also be eliminated. In the first place, the sex distribution of the disorder is in the opposite direction to that expected on a sex-linked 
recessive hypothesis, for females are affected by MS more frequently than males. A possibility is sex-linked dominance, with partial lethality of the hemizygotes, so that a disproportionate number of males are eliminated before they reach the age of being affected. For this there is no evidence at all: if anything the sex ratio in the sibships shows a male excess and again the pedigrees are not compatible with this hypothesis.

\section{Polygenic inheritance}

The alternative polygenic hypothesis is much more promising. The decline in frequency of affected relatives, the more remote the degree of relationship in the families of propositi; the elevated incidence of affected relatives in families of propositi compared with families of controls; the consistent excess of affected females: all these are compatible with a multifactorial aetiology of MS, the genetic component being polygenic. The only feature that does not support this hypothesis is the lack of elevation of frequency in relatives of the male propositi, the less frequent sex (they occur in the families of five male and five female patients) but for this again small population size may be responsible. The attempt to calculate the heritability of MS on this hypothesis therefore appears reasonable. It is unfortunate that despite the comprehensive nature of the survey the numbers remain too few for a satisfactory answer, particularly since the tentative estimates that emerge appear to be slightly lower than the remarkably consistent figures from other surveys (Table 2) which range from $40 \%$ to $48 \%$ in estimates using parents and from $56 \%$ to $57 \%$ using sibs (McAlpine et al., 1972). But from them the estimate of the genetic component in liability to develop MS can be appraised comparatively. It appears to be similar to, perhaps slightly greater than, that in diabetes $\left(h^{2}=35 \%\right.$ : Falconer, 1967), or in adult peptic ulcer $\left(h^{2}=37 \%\right.$ : Falconer, 1965) but certainly less than in pyloric stenosis $\left(h^{2}=79 \%\right.$ : Falconer, 1965), in cleft lip and palate $\left(h^{2}=65 \%-80 \%\right.$ : Bear, 1976), or in schizophrenia (60\%-90\%: Gottesman and Shields, 1968).

All the recent findings are concordant with the multifactorial hypothesis, which does not preclude environmental components such as possible viral infection or diet, and is compatible with the gene marker associations. The association with each allele or haplotype so far postulated is to be interpreted as indicating that that allele is merely one in the polygenic system, and so essentially contributes to the loading for susceptibility, rather than determines it. The reason why so many patients do not have HLA-B7 or DW2 is that they have a sufficient loading of other contributing polygenes in the

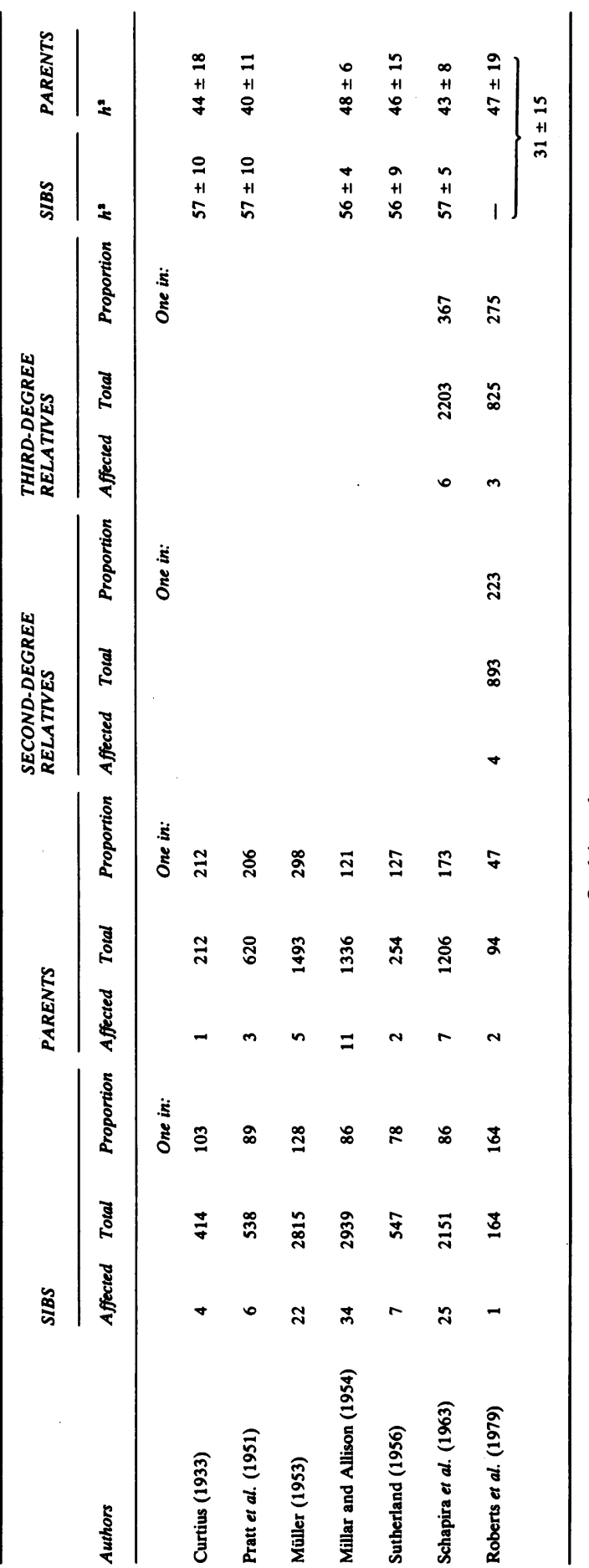

Table 2 Incidence of affected relatives 
system, and the varying strength of the association with the histocompatibility loci in different surveys is explicable in terms of the differing importance of the polygenes at other loci. In a relatively closed and long-established population such as Orkney, these polygenes would be widely distributed through the population, and their independent assortment would give rise to the pattern of familial aggregation as seen despite the intense interlocking of the ancestry of affected and unaffected individuals.

\section{Conclusion}

This analysis of MS in a total relatively compact population, the first to apply modern methods of population genetics to help elucidate an aetiological problem, although time-consuming, has produced some rewarding suggestions. It suggests that there may be a genetic component in MS but that this is not simple. Monogenic inheritance in any form is unlikely, unless there is greatly reduced penetrance and gross environmental interference. Much more likely is a complex aetiology, the genetic contribution being polygenic and possibly subordinate to the environmental, and with this hypothesis all recent observations can be reconciled.

We thank Mrs. J. A. Cowie and her associates; Dr. J. C. Bear; Mrs. Katherine Kemp; and the staff of Register House, Edinburgh.

This study was supported by the National Institute of Neurological and Communicative Disorders and Stroke (contracts NIH-NINCDS NO1-NS-4-2321 and NO1-NS-6-2337) and the Multiple Sclerosis Society of Great Britain and Northern Ireland.

Reprints from Professor D. F. Roberts, Department of Human Genetics, University of Newcastle upon Tyne, Newcastle upon Tyne NE1 7RU.

\section{References}

Bear, J. C. (1976). A genetic study of facial clefting in northern England. Clinical Genetics, 9, 277-284.
Bell, J. (1940). A determination of the consanguinity rate in the general hospital population of England and Wales. Annals of Eugenics, London, 10, 370-391.

Curtius, F. (1933). Multiple Sklerose und Erbanlage. Leipzig: Thieme.

Falconer, D.S. (1965). The inheritance of liability to certain diseases estimated from the incidence among relatives. Annals of Human Genetics, 29, 51-76.

Falconer, D. S. (1967). The inheritance of liability to disease with variable age of onset, with particular reference to diabetes mellitus. Annals of Human Genetics, 31, 1-20.

Gottesman, I. I. and Shields, J. (1968). In pursuit of the schizophrenic genotype. In Progress in Human Behaviour Genetics, pp. 67-103. Edited by S. G. Vandenberg. Johns Hopkins: Baltimore.

McAlpine, D., Lumsden, C. E., and Acheson, E. D. (Eds.) (1972). Multiple Sclerosis (second edition). Livingstone: London.

Masterson, J. C. (1973). Cousin marriage in Ireland. In Genetic Variation in Britain, pp. 59-66. Edited by D. F. Roberts and E. Sunderland. Taylor and Francis: London.

Millar, J. H. D., and Allison, R. S. (1954). Familial incidence of disseminated sclerosis in Northern Ireland. Ulster Medical Journal, 23, 29-91.

Morton, N. E., and Hussels, I. (1970). Demography of inbreeding in Switzerland. Human Biology, 42, 65-78.

Morton, N. E., Harris, D. E., Yee, S., and Lew, R. (1971). Pingelap and Mokil Atolls: migration. American Journal of Human Genetics, 23, 339-349.

Muller, R. (1953). Genetic aspects of multiple sclerosis. Archives of Neurology, 70, 733-740.

Myrianthopoulos, N. C., and MacKay, R. P. (1960). Multiple sclerosis in twins and their relatives. Genetic analysis of family histories. Acta genetica et statistica medica, 10, 33-47.

Poskanzer, D. C., Terasaki, P. I., Park, M. S., Prenneý, L. B., and Sheridan, J. L. (1980). Multiple sclerosis in Orkney and Shetland, I. Histocompatibility determinants. (In preparation).

Pratt, R. T. C., Compston, N. D., and McAlpine, D. (1951). The familial incidence of disseminated sclerosis and its significance. Brain, 74, 191-232.

Roberts, D. F. (1969). Consanguineous marriages and calculation of the genetic load. Annals of Human Genetics, 32, 407-410.

Roberts, D. F., Papiha, S. S., and Poskanzer, D. C. (1979) Polymorphisms and multiple sclerosis in Orkney. Journal of Epidemiology and Community Health, 33, 236-242.

Schapira, K., Poskanzer, D. C., and Miller, H. (1963). Familial and conjugal multiple sclerosis. Brain, 86, 315-332.

Sutherland, J. M. (1956). Observations on the prevalence of multiple sclerosis in Northern Scotland. Brain, 79, 635-654. 